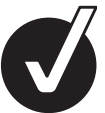

EDITOR'S

CHOICE

\title{
Biomarkers of ambient air pollution and lung cancer: a systematic review
}

\author{
Christiana A Demetriou, ${ }^{1,2}$ Ole Raaschou-Nielsen, ${ }^{3}$ Steffen Loft, ${ }^{4}$ Peter Møller, ${ }^{4}$ \\ Roel Vermeulen, ${ }^{5}$ Domenico Palli, ${ }^{6}$ Marc Chadeau-Hyam, ${ }^{1}$ Wei W Xun, ${ }^{1}$ Paolo Vineis ${ }^{1}$
}

- Additional materials are published online only. To view these files please visit the journal online (http://dx.doi.org/ 10.1136/oemed-2011-100566)

${ }^{1}$ Department of Epidemiology and Biostatistics, Imperial College London, London, UK ${ }^{2}$ Department of Electron Microscopy and Molecular Pathology, The Cyprus Institute of Neurology and Genetics,

Nicosia, Cyprus

${ }^{3}$ Institute of Cancer

Epidemiology, Danish Cancer

Society, Copenhagen, Denmark

${ }^{4}$ Section of Environmental

Health, Department of Public

Health, University of

Copenhagen, Copenhagen,

Denmark

${ }^{5}$ Institute for Risk Assessment

Sciences, Utrecht University,

Utrecht, the Netherlands

${ }^{6}$ Molecular and Nutritional Epidemiology Unit, Cancer

Research and Prevention Institute-ISPO, Florence, Italy

\section{Correspondence to}

Christiana A Demetriou Department of Electron Microscopy and Molecular Pathology The Cyprus Institute of Neurology and Genetics PO Box. 23462, 1683 Nicosia Cyprus; christiana.

demetriou08@imperial.ac.uk

Accepted 10 March 2012

Published Online First

7 July 2012

\section{ABSTRACT}

The association between ambient air pollution exposure and lung cancer risk has been investigated in prospective studies and the results are generally consistent, indicating that long-term exposure to air pollution may cause lung cancer. Despite the prospective nature and consistent findings of these studies, causality assessment can benefit from biomarker research. In the present systematic review, we assess the contribution of intermediate biomarkers in epidemiological studies, to ascertain whether their measurement reinforces causal reasoning. We have reviewed 524 papers which described the relationships between ambient air pollution and biological markers of dose and early response. The evidence for each marker was evaluated using assessment criteria which rate a group of studies from $A$ (strong) to $C$ (weak) on amount of evidence, replication of findings, and protection from bias. Biomarkers that scored A or B for all three criteria are included here. The markers that fulfilled the inclusion criteria are: 1-hydroxypyrene, DNA adducts, chromosomal aberrations, micronuclei, oxidative damage to nucleobases, and methylation changes. These biomarkers cover the whole spectrum of disease onset and progression from external exposure to tumour formation and some have also been suggested as risk predictors of future cancer, reinforcing causal reasoning. However, methodological issues such as confounding, publication bias and use of surrogate tissues instead of target tissues in studies on these markers are of concern. The identified biological markers have potential to shed light on the pathways of carcinogenesis, thus defining the association more clearly for public health interventions.

\section{AIR POLLUTION AND LUNG CANCER: STRENGTH OF EVIDENCE}

The association between exposure to ambient air pollution and the risk of lung cancer has been evaluated in a number of prospective studies, which are summarised in supplementary online table 1 . The evidence linking exposure to urban air pollutants, mainly particulate matter $\left(\mathrm{PM}_{2.5}\right.$ or $\left.\mathrm{PM}_{10}\right)$, with lung cancer is generally consistent, albeit formal statistical significance was not always reached. Cohorts from the USA as well as from Europe demonstrated increased risks of lung cancer with higher exposure to PM and other substances present in polluted air, with statistically significant risk ratios ranging from 1.14 to 5.21 (see supplementary online table 1 for references).

The main strength of the studies above resides in their prospective nature, with exposure being assessed long before disease ascertainment. However, causality is still uncertain, as a recent document by the Health Effects Institute has stressed. ${ }^{1}$ In the present systematic review we evaluate the contribution of biological markers of internal dose, biologically effective dose, and early effect in epidemiological studies on air pollution, to ascertain whether such contribution reinforces causal reasoning.

Measurement of biological markers of dose and effect can improve investigation of the health effects of various exposures, including air pollution, by facilitating improved exposure assessment and increased understanding of mechanisms, thereby providing biological plausibility, and investigation of individual susceptibility. ${ }^{2}$

This review aims to identify biological markers of dose and effect for which there is consistent evidence in the literature, to support the results of epidemiological studies on the effects of ambient air pollution. Epidemiological evidence from the selected studies has been assessed using a set of criteria that have been developed elsewhere. ${ }^{3}$ These account for (1) the total number of subjects investigated, (2) the degree of replication of findings across studies, and (3) potential protection from bias and/or confounding. PRISMA guidelines were also used to structure the analyses and to report the results. ${ }^{4}$

\section{METHODS}

\section{Search strategy and selection criteria}

Online databases PUBMED and OvidSP were searched to identify papers that evaluated the effects of ambient air pollution using biological markers up to January 2012. This search encompassed studies on subjects who have been exposed to environmental air pollution at their place of residence or at work, including traffic related air pollution. As illustrated in online supplementary figure $1,^{5}$ search terms included 'ambient' and 'traffic-related air pollution', 'particulate matter', 'polycyclic aromatic hydrocarbons', 'benzene', ' $\mathrm{NO}_{\mathrm{x}}$ ', and ' $\mathrm{SO}_{\mathrm{x}}$ '. References within each paper found during the initial search were also investigated and relevant papers identified. The resulting papers evaluated exposure using a variety of methods: personal air sampling, ambient pollution data from monitoring sites close to the place of residence or workplace, or traffic density in the place of residence. Only papers published in English were reviewed. The final reference list was based on relevance to the broad scope of this review, with papers without relevant exposure or outcome, 
studies on animals or in vitro studies, and perspectives and opinion reviews all excluded.

Papers were categorised according to the type of biological marker under investigation. As illustrated in figure $1,{ }^{6}$ biomarkers can reflect each step in a causal pathway from exposure to disease. They are usually grouped as biomarkers of: (1) internal dose; (2) biologically effective dose, indicating how much the exposure has damaged the molecules in the body and has possibly been removed by metabolic or repair mechanisms; (3) biological effects indicating changes in function or permanent alterations; (4) disease; and (5) susceptibility, which can modify transition rates at each step. Based on the figure, the biomarkers in this review were defined as biological markers of: (1) internal dose, which included 1-hydroxypyrene (1-OHP); (2) effective dose, which included DNA adducts and oxidised nucleobases; and (3) early effect, which included chromosomal aberrations (CAs), sister chromatid exchanges (SCEs) and micronuclei (MN), as well as mutations in the Hypoxanthine phosphoribosyltransferase (HPRT) gene and changes in methylation patterns. As explained in the Discussion section, we have not examined markers of genetic susceptibility related to gene variants or markers of inflammation. Figure 1 also shows the location of each of these biological markers in the pathway to disease. The response and step transition time can vary at each step with half-lives of, for example, 1-OHP, oxidised nucleobases and gene expression counted in hours, whereas bulky adducts show half-lives of weeks and for CAs and MN the halflife can be years. In lung cancer pathogenesis, the central mechanisms are considered damage to DNA in the form of bulky adducts and base oxidation from biotransformed polycyclic aromatic hydrocarbons (PAHs) and oxidative stress, as well as inflammation, with resulting chromosome damage and mutations. These changes, together with altered gene regulation, can lead to loss of cell cycle control and genomic instability. ${ }^{2}$

\section{Evaluation criteria}

For each biological measure, epidemiological evidence from the corresponding papers was assessed by generalising the Venice criteria, which were initially developed in the context of genetic association studies. ${ }^{3}$ These criteria are based on a scoring strategy according to three characteristics: (1) amount of evidence (sample size); (2) results replication; and (3) protection from potential bias and/or confounding. As detailed in table 1, biological markers of dose and effect with a large amount of evidence (total sample size $>1000$ ) were scored with A for amount of evidence. ${ }^{3}$ Similarly, markers extensively replicated among studies scored A for replication, provided that at least one well conducted meta-analysis with limited between-study heterogeneity was available. However, meta-analyses being rare in this field, some markers were scored A only on the basis of clear result replication (ie, unambiguous agreement in showing or not showing a significant association). Biological markers were finally scored A for protection from bias if potential bias could affect the magnitude but not the presence of the association, with $B$ if there was no obvious bias that could affect the presence of the association but there was considerable missing information concerning possible bias, and with $\mathrm{C}$ if the studies demonstrated potential for bias that could affect the presence or absence of the association. Confounding and publication bias are two important limitations of the studies we assessed, to which we have devoted a specific section in this review. In particular, we have assessed publication bias separately at the end of the Results section. The analytical methodology as well as reporting were based on the PRISMA guidelines.

It is recognised that the studies are heterogeneous within the specific exposure circumstances that they evaluate, and this may contrast with the application of a single score for the assessment of causality. However, the general exposure studied in this review is ambient air pollution and all the reviewed studies can be grouped under this broad category.

In the present review, only biological markers of internal dose, biologically effective dose and early effect that scored $\mathrm{A}$ or $\mathrm{B}$ for all three criteria are included: 1-OHP, DNA adducts, CAs, MN, oxidised nucleobases and methylation changes. SCEs and HPRT mutations were not considered further as they failed to score A or B for the three criteria. Data from relevant studies were extracted and are summarised in online supplementary tables 1-10. Quantitative meta-analyses were not performed, owing to the large heterogeneity between the included studies. However, for clarity, the results for DNA adducts, oxidised nucleobases and CAs were summarised as standard mean differences $(\mathrm{SMD})$ in forest plots.

We have focused on genotoxic and epigenotoxic effects as markers of biologically effective dose and biological effect directly related to carcinogenesis. Although chronic inflammation is considered relevant to particle-induced lung carcinogenesis, $^{7}$ at least one mechanism of action is thought to involve oxidative stress-induced DNA damage, which is addressed here. ${ }^{8}$ While exposure to air pollutants has been associated with acute inflammation in the airways and to elevated levels of systemic markers of inflammation, such as $\mathrm{C}$ reactive protein and

Figure 1 Biological markers of exposure and effects of air pollution. Adapted from Loft et al. ${ }^{5}$ 
Table 1 The grading criteria for the evaluation of cumulative evidence on the relationship between air pollution and biomarkers

\begin{tabular}{|c|c|c|}
\hline Criteria & Categories & Proposed operationalisation \\
\hline Amount of evidence & $\begin{array}{l}\text { A: Large-scale evidence } \\
\text { B: Moderate amount of evidence } \\
\text { C: Little evidence }\end{array}$ & $\begin{array}{l}\text { Thresholds may be defined based on sample size, power or false-discovery } \\
\text { rate considerations. As a simple rule, we suggest that category A requires } \\
\text { a sample size of over } 1000 \text { (total number in cases and controls assuming } \\
1: 1 \text { ratio) evaluated in the least common genetic group of interest; } \\
\text { B corresponds to a sample size of } 100-1000 \text { evaluated in this group, and } \\
\text { C corresponds to a sample size of }<100 \text { evaluated in this group. }\end{array}$ \\
\hline Replication & $\begin{array}{l}\text { A: Extensive replication including at least one well-conducted } \\
\text { meta-analysis with little between-study inconsistency } \\
\text { B: Well-conducted meta-analysis with some methodological } \\
\text { limitations or moderate between-study inconsistency } \\
\text { C: No association; no independent replication; failed replication; } \\
\text { scattered studies; flawed meta-analysis or large inconsistency }\end{array}$ & $\begin{array}{l}\text { Between-study inconsistency entails statistical considerations (eg, defined } \\
\text { by metrics such as } \mathrm{I}^{2} \text {, where values of } 50 \% \text { and above are considered large, } \\
\text { and values of } 25 \%-50 \% \text { are considered moderate inconsistency) and also } \\
\text { epidemiological considerations for the similarity/standardisation or at least } \\
\text { harmonisation of phenotyping, genotyping and analytical models across studies. }\end{array}$ \\
\hline Protection from bias & $\begin{array}{l}\text { A: Bias, if at all present, could affect the magnitude but probably } \\
\text { not the presence of the association } \\
\text { B: No obvious bias that may affect the presence of the association, } \\
\text { but there is considerable missing information on the generation of } \\
\text { evidence } \\
\text { C: Considerable potential for or demonstrable bias that can affect } \\
\text { even the presence or absence of the association }\end{array}$ & $\begin{array}{l}\text { A prerequisite for A is that the bias due to phenotype measurement, genotype } \\
\text { measurement, confounding (population stratification) and selective reporting } \\
\text { (for meta-analyses) can be appraised as not being high plus there is no other } \\
\text { demonstrable bias in any other aspect of the design, analysis or accumulation } \\
\text { of the evidence that could invalidate the presence of the proposed association. } \\
\text { In category B, although no strong biases are visible, there is no such assurance } \\
\text { that major sources of bias have been minimised or accounted for because } \\
\text { information is missing on how phenotyping, genotyping and confounding } \\
\text { have been handled. Given that occult bias can never be ruled out completely, } \\
\text { note that even in category A, we use the qualifier 'probably'. }\end{array}$ \\
\hline
\end{tabular}

Adapted from loannidis et al. Int J Epidemiol 2008;37:120-32 (See supplementary file for references).

fibrinogen, this has so far mainly been associated with the risk of cardiovascular diseases. ${ }^{9}$

\section{RESULTS}

Biological markers of exposure and internal dose: 1-0HP

1-OHP is a useful marker for occupational exposure and has also become the biomarker most commonly used to assess exposure to traffic-related air pollution and particularly to PAHs. It is a urinary excreted metabolite of pyrene and can be measured as a marker of systemic absorption of PAHs. ${ }^{10} 11$

Based on our inclusion criteria, eight papers and one review studied the association between exposure to air pollution or chemicals in polluted air and the levels of 1-OHP excretion in the urine of exposed individuals. Online supplementary tables 2 and 3 summarise the associations reported in these studies. Some of the studies suggested positive associations in adults, for example, mail carriers and bus drivers, ${ }^{12-14}$ and other studies showed higher 1-OHP levels in exposed children. ${ }^{15-19}$

\section{Confounding}

Among the studies on 1-OHP, five adequately adjusted for confounders including smoking data, where relevant. ${ }^{13}$ 15-17 19 One of the studies only adjusted for smoking, ${ }^{14}$ one did not mention confounding ${ }^{18}$ and one was a review. ${ }^{12}$

\section{Grading}

The 1-OHP information was graded A for evidence, A for replication and $B$ for bias. Although the overall number of subjects is large $(\mathrm{N}=1708)$ and findings have been replicated several times, it is not completely clear whether confounding from smoking, occupational exposures or environmental tobacco smoke can be ruled out, which justifies a B for the third grading criterion

Biological markers of exposure and effective dose: DNA adducts DNA adducts are formed when carcinogens, or metabolites of carcinogens, react with sites in DNA, resulting in the formation of a covalent bond between the carcinogen and DNA. Even though adducts can be removed by repair proteins, some can persist. This can result in nucleotide substitutions, deletions and chromosome rearrangements during replication, contributing to cancer development. ${ }^{20}$ Numerous studies have considered DNA adducts as a biomarker of exposure to genotoxic carcinogens. The studies reported here $(\mathrm{N}=25)$ are cross-sectional and casecontrol studies, some of which were nested in prospective cohorts. Some studies carried out correlation and regression analyses on all subjects (online supplementary table 4), while others compared the mean DNA adduct levels in individuals with estimated high or low external exposures (online supplementary table 5). As illustrated in figure 2, most studies (including two reviews) suggested positive associations between exposure to air pollution or chemicals in polluted air and the formation of DNA adducts in exposed individuals. Subjects in these studies included, among others, policemen in Bangkok, ${ }^{14}$ schoolchildren in Thailand, ${ }^{17}$ policemen in Genoa ${ }^{21}$ and in Prague, ${ }^{22}$ residents in an industrial area and rural controls in Poland, ${ }^{23}$ bus and taxi drivers in Stockholm, ${ }^{24}$ bus drivers in Copenhagen, ${ }^{25}$ students in Denmark and in Greece, ${ }^{26}$ as well as street vendors, taxi drivers, gasoline salesmen and roadside residents in Benin. ${ }^{27}$ Fetal exposures and DNA adducts in newborns also showed positive associations. $^{28-30}$ Only two studies reported no association. ${ }^{31} 32$

\section{Confounding}

Of 14 studies investigating DNA adducts which adjusted for a number of confounders, seven adjusted for PAHs in diet. One study adjusted only for smoking, and one only for various risk alleles. For six studies there is no information on confounding. Two publications were reviews and for one study confounding was not mentioned by the authors as the measurements were from the same subjects before and after a change in working conditions, within a 3-month interval, during which exposure to potential confounder(s) can be assumed to be constant (online supplementary table 6a).

\section{Grading}

The DNA adducts information was graded A for evidence, A replication and $B$ for bias. The association between ambient air pollution and DNA adducts has been shown in a large number of subjects $(\mathrm{N}=3075)$ and replicated. Confounding is unlikely in the studies that included only never and ex-smokers such as 


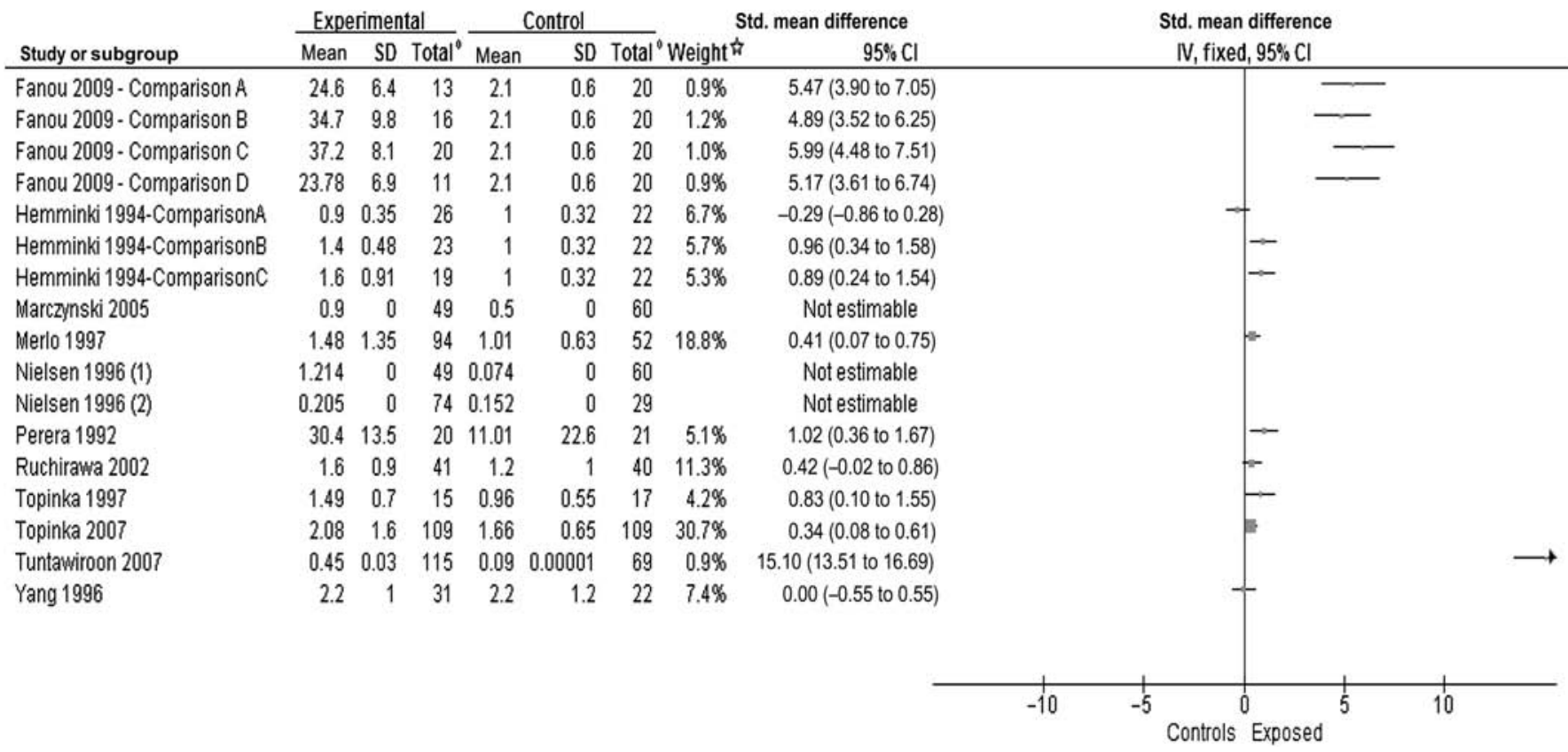

Figure 2 Standardized mean difference forest plot of studies on DNA adducts reporting difference in means. Weight was derived using the inverse of the variance in a fixed effects model. Forest plots are presented for clarity in data presentation. However, formal meta-analysis was not performed due to the heterogeneity of the studies included in the review. Total refers to total sample size in the experimental (exposed) and control groups (See supplementary file for references).

Peluso et al. ${ }^{33}$ However, publication bias cannot be entirely excluded (see Publication bias and heterogeneity section below) and a major determinant of DNA adducts is $\operatorname{diet}^{34}$ (which was not ascertained in most studies), so we rate $\mathrm{B}$ for the third grading criterion. It is important to mention that the levels of DNA adducts in white blood cells (WBCs) have been shown to predict the risk of lung cancer in cohort studies and recently in a prospective pooled analysis. ${ }^{35}$

\section{Biological markers of exposure and effective dose: oxidised nucleobases}

More data are available ( $\mathrm{N}=34$ publications) concerning oxidised nucleobases to nucleobases as this is one of the most plausible mechanisms by which air pollutants may affect lung pathophysiology (online supplementary figure 2). Oxidised nucleobases refers to modified purine and pyrimidine bases formed when reactive oxygen species (ROS) react with DNA or the nucleotide pool. Substances such as PM can generate ROS directly or through enzymatic reactions in target or inflammatory cells ${ }^{36}$; ozone and $\mathrm{NO}_{2}$ are themselves reactive species, and benzene metabolism can also generate ROS. ${ }^{10}$ Oxidatively modified DNA bases have the potential to damage the integrity of the genome. For example, 8-oxo-7,8-dihydroguanine (8oxoGua), one of the most critical lesions, leads to GC to TA transversion unless repaired prior to DNA replication. Cell levels of 8-oxoGua are usually measured as its 2'-deoxyribonucleoside equivalent 8-oxo-7,8-dihydro-2' -deoxyguanosine (8-oxodG). The most relevant repair in terms of base excision results in 8oxoGua, which, however, is difficult to measure in urine, whereas 8-oxodG resulting from other putative repair pathways and nucleotide pool sanitisation can be readily calculated. Lastly, formamidopyrimidine DNA glycosylase (FPG) sensitive sites in mononuclear blood cells (MNBC) are a marker of oxidative damage. FPG is a base excision repair enzyme which recognises and removes oxidised purines, including 8-oxoGua.
The effects of air pollution on oxidised nucleobases have been studied in controlled exposure scenarios, in panel and in crosssectional studies (online supplementary tables 6 and 7). The results of studies comparing mean levels of markers of guanine oxidation (8-oxodG or 8-oxoGua) are summarised in a forest plot (figure 3) which illustrates that for the majority of studies, biomarker levels are higher in exposed subjects compared to controls (positive SMD).

The effect of controlled exposure to air pollution (mainly traffic generated ultrafine particles with diameter $<100 \mathrm{~nm}$ ) has been investigated in healthy humans showing usually a higher level of FPG sensitive sites in MNBC in the exposed subjects than in the unexposed. ${ }^{37-39}$ In addition, increased urinary excretion of 8-oxoGua was observed in studies where subjects were exposed to exhaust in traffic-intense areas. ${ }^{40} 41$

A number of panel studies which have also investigated the effects of air pollution in the general population showed contradictory effects of air pollution on oxidised nucleobases. ${ }^{31} 4243$

The cross-sectional studies investigating the effect of air pollution on oxidised nucleobases can be grouped into two main categories according to their design. A first group of studies investigated the effect of air pollution among subjects with different occupational exposures. We refer here only to investigations in which the occupational exposure was qualitatively similar to the exposure of the general population (eg, we excluded categories with special exposures such as gasoline workers). Using job titles as the basis for stratification of exposure, subjects characterised by having jobs with high exposure to traffic emissions showed increased levels of oxidised nucleobases. A second group of residential studies generally showed positive associations between living and/or working in highly polluted areas and oxidised nucleobases. Studies using benzene as a marker of urban air pollution exposure also showed associations with markers of oxidised nucleobases. ${ }^{27}$ 44-47 


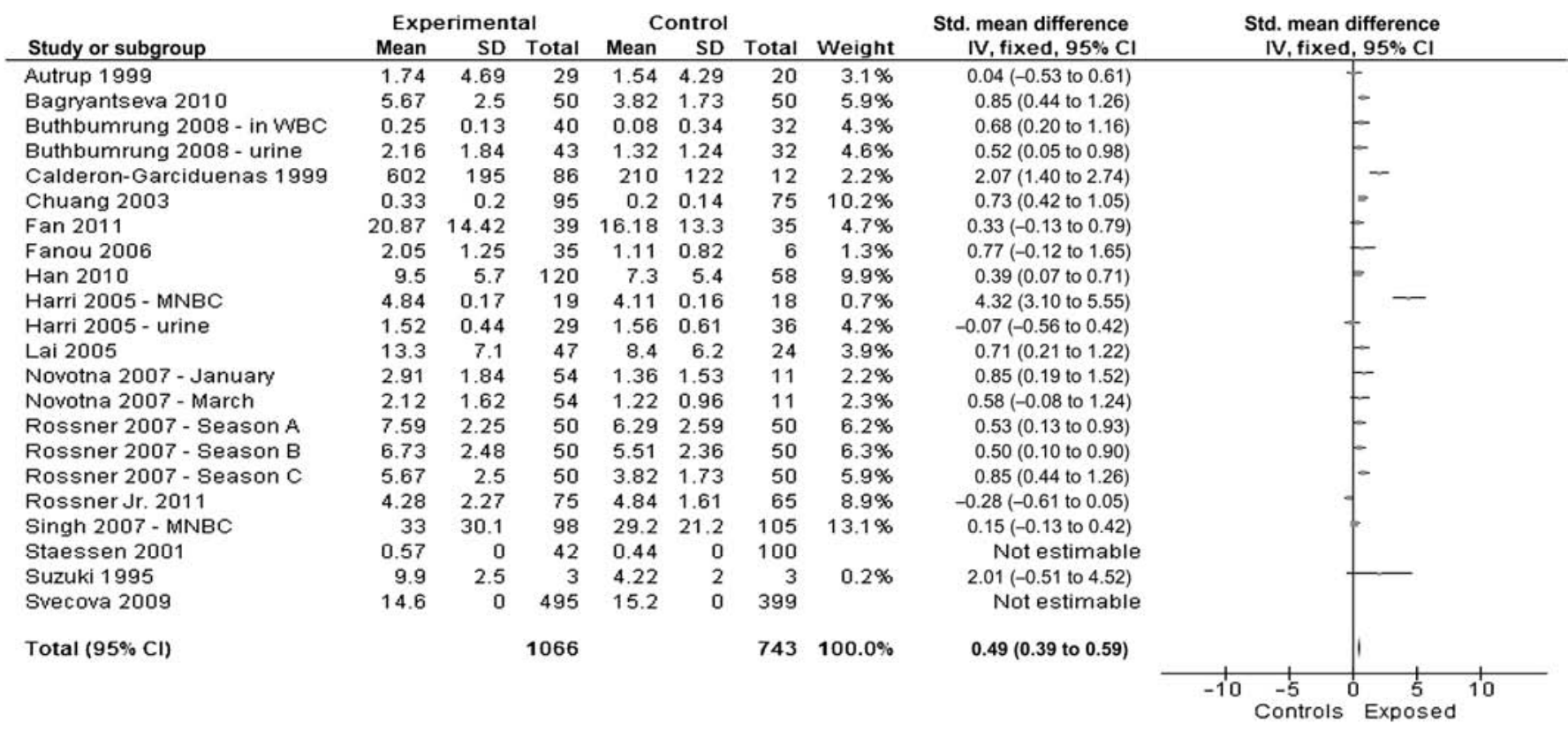

Figure 3 Standardised mean difference forest plot of studies on oxidised nucleobases reporting difference in means of 8-0xo-7,8-dihydro-2 deoxyguanosine or 8-0xo-7,8-dihydroguanine. Weight was derived using the inverse of the variance in a fixed effects model. Forest plots are presented for clarity in data presentation. Total refers to total sample size in the experimental (exposed) and control groups (See supplementary file for references).

A formal meta-analysis of the effects of air pollution on DNA base oxidation (measured in $\mathrm{MNBC}$ ) and excretion of repair products in urine, as well as an integrated analysis incorporating the endpoints of oxidatively damaged nucleobases in cultured cells, experimental animal models and humans, has been carried out. ${ }^{48}$ Despite large heterogeneity between studies, the analysis showed highly significant effects with an SMD between exposed and unexposed subjects of 0.53 in blood $(95 \%$ CI 0.29 to 0.76 ) and 0.52 in urine (0.22 to 0.82$)$. Based on the studies included in the current review, we have replicated these findings (results not shown).

\section{Confounding}

Among publications on oxidised nucleobases $(\mathrm{N}=34), 23$ adjusted for a number of confounders including smoking. Five studies adjusted only for metabolic genes and four studies were cross-over studies in short time frames, and confounding was therefore not relevant. For two studies there is no mention of confounders (online supplementary table 7a).

\section{Grading}

The oxidised nucleobases information was graded A for evidence, A for replication and A/B for bias. Altogether, there is consistent and strong evidence that exposure to ambient air pollution leads to increased levels of biomarkers of oxidation damage to nucleobases, both in observational and experimental studies. High urinary excretion of 8-oxodG or 8-oxoGua has been associated with increased risk of lung cancer in one prospective and several case-control studies. ${ }^{6}$

\section{Biological markers of early effect: CAs}

CAs are defined as modifications of the normal chromosome complement due to deletion, duplication or rearrangement of genetic material.

The studies on CAs $(\mathrm{N}=10)$ (online supplementary table 8) are not all supportive of a positive association with exposure to air pollution or its constituents in adults. As illustrated in figure 4 , some studies found a higher frequency of CAs with exposure to heavy air pollution, ${ }^{50-56}$ others did not find statistically significant associations ${ }^{57} 58$ and others produced contradictory results. $^{59}$

\section{Confounding}

Only six of the studies investigating CAs have adequately adjusted for confounders such as age, sex and smoking habits. ${ }^{50-53} 5558$ Three did not adequately adjust as they controlled only for age or only for sex. ${ }^{5456}$ One study did not mention adjustment for any confounders. ${ }^{57}$

\section{Grading}

The information on chromosome aberration was graded $\mathrm{A}$ for evidence, $\mathrm{B}$ for replication and B/C for bias. Even though not all studies agree, there is some evidence to support the association between exposure to air pollution and chromosome aberration frequencies. Confounding and publication bias cannot be ruled out.

\section{Biological markers of early effect: MN}

$\mathrm{MN}$ are nuclei, separate from and additional to the main nucleus of a cell. During cell division, DNA replicates and divides equally between the two daughter cells. If the process is disrupted, or the chromosomes are broken or damaged by chemicals or radiation, then the distribution of genetic material between the two daughter nuclei may be affected and pieces or entire chromosomes may fail to be included in either of the two daughter nuclei. The genetic material that is not incorporated into a new nucleus may form its own 'micronucleus' ${ }^{60}$ Thus, MN are a marker of chromosomal damage.

Four recent studies ${ }^{21566162}$ and a review ${ }^{63}$ have looked at the association between ambient air pollution or its constituents, and $\mathrm{MN}$ in the cells of exposed individuals (online supplementary table 9), finding positive associations.

\section{Confounding}

There is one review and four studies on $\mathrm{MN}$, two of which have adjusted for smoking and gender, ${ }^{61} 62$ and one included some 


\begin{tabular}{|c|c|c|c|c|c|c|c|c|c|c|}
\hline \multirow[b]{2}{*}{ Study or subgroup } & \multicolumn{3}{|c|}{ Experimental } & \multicolumn{2}{|c|}{ Control } & \multirow[b]{2}{*}{ Total } & \multirow[b]{2}{*}{ Weight } & Std. mean difference & \multirow{2}{*}{$\begin{array}{l}\text { Std. mean difference } \\
\text { IV,Fixed, } 95 \% \mathrm{Cl}\end{array}$} & \\
\hline & Mean & SD & Total & Mean & SD & & & IV,Fixed, $95 \% \mathrm{Cl}$ & & \\
\hline Balachandar 2008 & 5 & 1.68 & 18 & 1.16 & 0.92 & 18 & $1.4 \%$ & 2.77 (1.83 to 3.71$)$ & . & \\
\hline Burgaz 2002 & 1.82 & 0.34 & 29 & 0.26 & 0.14 & 5 & $0.5 \%$ & 4.73 (3.21 to 6.26$)$ & 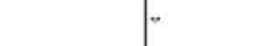 & \\
\hline Burgaz 2002- Comparison B & 1.29 & 0.3 & 18 & 0.26 & 0.14 & 5 & $0.5 \%$ & 3.59 (2.08 to 5.10$)$ & 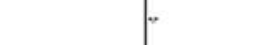 & \\
\hline Knudsen 1999 & 2.84 & 1.87 & 55 & 2.24 & 1.57 & 45 & $7.9 \%$ & $0.34(-0.06$ to 0.74$)$ & & \\
\hline Knudsen 1999-ComparisonB & 2.12 & 1.38 & 60 & 2.46 & 1.98 & 41 & $7.9 \%$ & $-0.20(-0.60$ to 0.19$)$ & & \\
\hline Kyrtopoulos 2001 & 0.88 & 0.97 & 222 & 1.06 & 1.12 & 149 & $28.9 \%$ & $-0.17(-0.38$ to 0.03$)$ & & \\
\hline Rossnerova 2011 & 0.8 & 0.27 & 86 & 0.61 & 0.21 & 92 & $13.4 \%$ & 0.79 (0.48 to 1.09$)$ & & \\
\hline Sram 1999 & 1.54 & 0 & 131 & 1.04 & 0 & 48 & & Not estimable & & \\
\hline Sram 2007 & 0.27 & 0.18 & 61 & 0.16 & 0.17 & 61 & $9.5 \%$ & $0.62(0.26$ to 0.99$)$ & & \\
\hline Zidzik 2007 -Comparison A & 2.6 & 2.64 & 51 & 2.14 & 1.61 & 55 & $8.6 \%$ & $0.21(-0.17$ to 0.59$)$ & & \\
\hline Zidzik 2007-Comparison B & 2.33 & 1.53 & 52 & 1.94 & 1.28 & 50 & $8.2 \%$ & $0.27(-0.12$ to 0.66$)$ & & \\
\hline Zidzik 2007-Comparison C & 3.04 & 1.64 & 50 & 1.79 & 0.77 & 45 & $6.9 \%$ & $0.95(0.53$ to 1.38$)$ & & \\
\hline Zidzik 2007-Comparison D & 3.6 & 1.63 & 50 & 1.79 & 0.77 & 45 & $6.2 \%$ & 1.38 (0.93 to 1.84$)$ & & \\
\hline \multirow[t]{2}{*}{ Total $(95 \% \mathrm{Cl})$} & & & 752 & & & 611 & $100.0 \%$ & 0.40 (0.29 to 0.51$)$ & & \\
\hline & & & & & & & & -100 & $0 \quad 50$ & $100^{\prime}$ \\
\hline
\end{tabular}

Figure 4 Meta-analyses: fixed effect model. Standardised mean difference forest plot of studies on chromosomal aberrations (CAs) reporting difference in means of CAs. Weight was derived using the inverse of the variance in a fixed effects model. Forest plots are presented for clarity in data presentation. However, formal meta-analysis was not performed due to the heterogeneity of the studies included in the review. Total refers to total sample size in the experimental (exposed) and control groups.

polymorphisms. ${ }^{62}$ Two studies only adjusted for sex. ${ }^{21} 56$ The study on newborns also adjusted for a number of relevant confounders. $^{61}$

\section{Grading}

The information on $\mathrm{MN}$ was graded A for evidence, B for replication and $\mathrm{B} / \mathrm{C}$ for bias. Given the replication of results between the studies, there is some evidence to support the association between exposure to air pollution and MN. However, confounding and publication bias cannot be entirely ruled out.

\section{Biological markers of early effect: methylation patterns}

DNA methylation refers to the addition of methyl groups to nucleotides. The genome has a well-established pattern of methylation. Increase or decrease of the methylated sites in DNA affects gene expression and can also lead to genomic instability. The degree of methylation is passed on to daughter strands at mitosis by maintenance DNA methylases. DNA methylation and the associated repressed or activated transcription of genes have been implicated in carcinogenesis. ${ }^{64}$ Five reports (from four studies) have recently investigated the effects of air pollution exposure on methylation patterns, ${ }^{30}$ 65-68 mostly focusing on long interspersed element-1 (LINE-1) and Alu elements methylation as measures of whole genome methylation (online supplementary table 10). LINE-1 and Alu elements are retrotransposons, repetitive and mobile sequences in the genome. LINEs make up a large proportion of the genome and LINE-1 as well as Alu methylation correlates with overall level of DNA methylation in the cell. LINE-1 methylation was frequently found to be altered by exposure to air pollution, ${ }^{65-68}$ and Alu methylation was also significantly altered in one study. ${ }^{68}$ One study investigated global methylation in cord blood samples with the use of an assay kit and found that it was altered in response to prenatal PAH exposure. ${ }^{30}$ These epigenetic changes can contribute to carcinogenesis at least as much as genetic changes.

\section{Confounding}

The five reports investigating methylation patterns have adequately adjusted for a number of clinical and environmental confounders, including smoking.

\section{Grading}

The information on methylation was graded as B for evidence, $\mathrm{B} / \mathrm{C}$ for replication and $\mathrm{B}$ for bias. The results above suggest that LINE-1 methylation levels may be affected by exposure to air pollution or its constituents. Even though only a few studies were available, the replication between them was fairly good, thus supporting the $\mathrm{B} / \mathrm{C}$ grading for replication. Alu methylation levels were less consistently affected. It is also relevant to note that LINE-1 methylation levels were found to increase with the level of exposure to some constituents of air pollution, for example $\mathrm{PM}_{10}$, but to decrease with exposure levels to other constituents such as $\mathrm{PM}_{2.5}$. Therefore, further evidence is needed to determine which constituents in air pollution affect methylation levels and in which direction, before we can more confidently draw conclusions about the effect of exposure to air pollution on methylation levels.

\section{Publication bias and heterogeneity}

One of the factors determining the third grading criterion is publication bias. As discussed, publication bias cannot be ruled out for most of the biological markers mentioned above. Funnel plots are a useful tool for checking the existence of publication bias, and a symmetric inverted funnel plot typically indicates that publication bias is unlikely. In this review, funnel plots could only be constructed for DNA adducts and oxidised nucleobases, where enough studies were available. Also, because of the diversity in effect estimates for each biological marker, only studies comparing mean levels of markers in cases and controls could be used. Moreover, different sample types (WBC, $\mathrm{MNBC}$ or urine), analytical methods and units were used for each marker. The funnel plots of SMDs (online supplementary 
figures 3 and 4) were fitted using a fixed effects model and using the inverse variance as weight. The asymmetrical inverted funnels thus obtained demonstrate that publication bias may be a concern when the available evidence on biological markers of dose and effect and the relationship with air pollution is investigated. However, despite the asymmetry in the plots, the Egger's regression asymmetry test did not demonstrate a significant presence of publication bias for studies on DNA adducts or oxidised nucleobases $(p=0.376$ and $p=0.576$, respectively).

\section{DISCUSSION}

On the basis of the evidence from recent large cohort studies in the USA and in Europe (online supplementary table 1), it has been suggested that ambient air pollution may increase lung cancer risk.

Overall, existing biological markers of dose and effect appear to reinforce the causal nature of the association between air pollution and lung cancer, although the markers in this review are not all specific to lung carcinogenesis. DNA adducts, CAs, $\mathrm{MN}$ and oxidised nucleobase markers have been suggested to be predictive for the risk of future cancer. ${ }^{35}$ The biological markers discussed in this review cover the whole spectrum of progression from external exposure to tumour formation (figure 1). 1-OHP is an excellent marker of internal dose, DNA adducts and oxidised nucleobases are markers of the biologically effective dose, while $\mathrm{MN}$, CA and DNA methylation are good markers of early biological effect. The multilevel evidence supports a causal association between exposure to ambient air pollution and lung cancer. The available evidence is stronger for oxidised nucleobase markers, and the mechanisms supported by these biological markers are likely to be central to the biological process of air pollution induced lung cancer (figure 1).

However, certain aspects of biological markers used in epidemiological studies need to be clarified. These include their reliability, the extent to which markers interact with genetic susceptibility, and inter-laboratory as well as inter-technique variation. Adequate adjustment for confounding factors needs to be considered. In the studies summarised above, body mass index, physical exercise, consumption of charcoal-broiled food, consumption of fresh fruits and vegetables, and seasonal variations were rarely controlled for (online supplementary tables $2-10)$. All these factors have been reported to influence bulky DNA adducts. Most studies have controlled for smoking, one of the most relevant confounders regarding exposure to air pollution and biological markers. Finally, the association between air pollution and biological markers of dose and effect depends on the level of exposure, with low levels of exposure often leading to weak and non-significant associations.

An issue difficult to tackle in studies utilising biological markers, which are usually small in size, is publication bias. Funnel plots (online supplementary figures 3 and 4 ) do not show extensive publication bias. However, there is some asymmetry of the plots and there are only few large studies showing positive effects, implying some bias (online supplementary tables 2-10).

We have focused on genotoxic and epigenotoxic effects as markers of biologically effective dose and biological effect directly related to carcinogenesis, while we have not included markers of inflammation. Although chronic inflammation is probably relevant to particle-induced lung carcinogenesis, the overall evidence is still relatively scanty. Exposure to air pollutants has been associated with acute inflammation in the airways and to elevated levels of systemic markers of inflammation, such as $\mathrm{C}$ reactive protein and fibrinogen. A recent study found that medium-term exposure to traffic-related air pollution may induce an increased inflammatory/endothelial response, especially among people with diabetes. ${ }^{69}$ So far the inflammatory response has mainly been associated with the risk of cardiovascular diseases rather than cancer. ${ }^{9}$

The main limitations we identified in our review are related to control of confounding and publication bias. In addition, almost none of the studies investigated more than one mechanistic pathway. Maybe the next generation of studies could address confounding in a more systematic way (eg, by measuring cotinine) and will include markers that refer to more than one pathway (eg, inflammation and epigenetics). Publication bias is a general problem in epidemiology and requires concerted action by journal editors.

Another important, and probably largely unavoidable, limitation of these studies is that they are based on surrogate tissues (eg, WBCs) that do not necessarily reflect changes in the target tissues. Due to the difficulties associated with obtaining lung tissue samples, surrogate tissues are used to estimate the damage caused in the target tissue. In the case of air pollution and lung cancer, lung tissue is the first point of contact with the carcinogen and therefore damage in this tissue is likely to be more pronounced than damage in surrogates such as WBCs.

In spite of methodological limitations, there is overall good evidence concerning the genotoxicity of air pollution. Applying grading criteria for causal assessment, we concluded that the cumulative evidence indicates that air pollution affects some of the biological markers related to carcinogenesis, particularly 1-OHP, DNA adducts and 8-oxodG and other oxidised nucleobases. Some markers of genotoxicity have also been found to be associated with lung cancer (DNA adducts and 8-oxodG/8oxoGua in urine). Lung cancer develops via a series of progressive pathological changes in the respiratory epithelium. Molecular alterations such as loss of heterozygosity, gene mutations and gene promoter methylation have emerged as mechanisms of lung carcinogenesis. ${ }^{2}$

Although information obtained from biomarkers adds to the knowledge obtained from prospective epidemiological studies on the effects of air pollution, the evidence overall is still incomplete and fragmented. Not only is the evidence for several markers still equivocal, but we are far from being able to reconstruct the full pathogenetic pathway that leads from external exposure to the outcome of lung cancer. Few studies have been conducted on epigenetic and non-genotoxic changes, so that the evidence is skewed in favour of genotoxicity biomarkers. We propose that future efforts should be directed not only towards reducing uncertainty concerning the role of specific biomarkers, but also towards filling the gaps in the supposed pathogenetic pathways.

\section{ADDITION TO SCIENTIFIC KNOWLEDGE AND CONCLUSIONS}

Our review evaluated the data available on some of the most relevant biomarkers of air pollution exposure, and used well accepted criteria to grade the cumulative evidence on each biomarker with respect to the amount of evidence, replication and protection from bias. Several biological markers of dose and effect related to carcinogenic mechanisms, and especially oxidised nucleobases, have been found to be associated with exposure to ambient air pollution, and some of these markers have also been associated with risk for lung cancer. ${ }^{35}$ These biological markers, which mark the continuum of progression from external exposure to cancer outcome, have the potential to shed light on the pathways of carcinogenesis, thus defining the association more clearly for public health interventions. 
To our knowledge, this is the first time a systematic evaluation of the topic has been undertaken. Our review adds biological support to the relationship between air pollution and lung cancer. Nonetheless, future research to fill gaps in our knowledge of supposed pathogenetic pathways is needed.

Acknowledgements The authors also acknowledge the TRANSPHORM, EU FP7 Grant agreement no. 243406.

Contributors $C A D$ wrote most sections of the manuscript, reviewed the whole document and is a guarantor; SL wrote the section on oxidative damage, contributed figures, and revised the manuscript critically for intellectual content; PM wrote the section on oxidative damage, contributed figures and revised the manuscript critically for intellectual content; OR-N, RV and MC-H presented ideas, provided critical comments on the conclusions and revised the manuscript critically for intellectual content; WX helped produce the funnel plots and revised the manuscript; PD revised the manuscript critically for intellectual content; and PV made a substantial contribution to the conception and design, and analysis and interpretation of data, revised the entire manuscript and is a guarantor.

Funding This work has been made possible by an EU grant to Dr Bert Brunekreef as part of the ESCAPE (European Study of Cohorts for Air Pollution Effects) project and TRANSPHORM, EU FP7 Grant agreement no. 243406 (start date: 1 June 2008, end date: 31 May 2011; MRC-HPA Centre for Environment and Health, Imperial College London).

\section{Competing interests None.}

Provenance and peer review Not commissioned; externally peer reviewed.

\section{REFERENCES}

1. Health Effects Institute. Traffic-Related Air Pollution: A Critical Review Of The Literature On Emissions, Exposure, And Health Effects. The Health Effects Institute: Boston, MA USA, 2010

2. Vineis $\mathbf{P}$, Husgafvel-Pursiainen K. Air pollution and cancer: biomarker studies in human populations. Carcinogenesis 2005;26:1846-55.

3. Ioannidis JP, Boffetta P, Little J, et al. Assessment of cumulative evidence on genetic associations: interim guidelines. Int J Epidemiol 2008;37:120-32.

4. Moher D, Liberati A, Tetzlaff J, et al. Preferred reporting items for systematic reviews and meta-analyses: the PRISMA statement. BMJ 2009;339:b2535.

5. Loft S, Møller P. Oxidative DNA damage and human cancer: need for cohort studies Antioxid Redox Signal 2006;8:1021-31.

6. Loft S, Svoboda P, Kasai H, et al. Prospective study of 8-oxo-7,8-dihydro-2'deoxyguanosine excretion and the risk of lung cancer. Carcinogenesis 2006;27:1245-50.

7. Borm PJA, Schins RPF, Albrecht C. Inhaled particles and lung cancer, part B: paradigms and risk assessment. Int J Cancer 2004;110:3-14.

8. Knaapen AM, Güngör N, Schins RPF, et al. Neutrophils and respiratory tract DNA damage and mutagenesis: a review. Mutagenesis 2006:21:225-36.

9. Frampton MW. Inflammation and airborne particles. Clin Occup Environ Med 2006;5:797-815

10. Loft S, Brauner E, Forchhammer L, et al. Complex exposures-air pollution. In: Wild C, Vineis P, Garte S, eds. Molecular Epidemiology of Chronic Diseases. West Sussex, England: Wiley, 2008:343-58

11. Jongeneelen FJ, Anzion RB, Leijdekkers CM, et al. 1-hydroxypyrene in human urine after exposure to coal tar and a coal tar derived product. Int Arch Occup Environ Health 1985; 57:47-55.

12. Castaño-Vinyals G, D'Errico A, Malats N, et al. Biomarkers of exposure to polycyclic aromatic hydrocarbons from environmental air pollution. Occup Environ Med 2004:61:e12

13. Hansen $\mathbf{A M}$, Wallin $\mathrm{H}$, Binderup ML, et al. Urinary 1-hydroxypyrene and mutagenicity in bus drivers and mail carriers exposed to urban air pollution in Denmark. Mut Res 2004;557:7-17.

14. Ruchirawa M, Mahidol C, Tangjarukij C, et al. Exposure to genotoxins present in ambient air in Bangkok, Thailand-particle associated polycyclic aromatic hydrocarbons and biomarkers. Sci Total Environ 2002;287:121-32.

15. Hansen AM, Raaschou-Nielsen 0, Knudsen LE. Urinary 1-hydroxypyrene in children living in city and rural residences in Denmark. Sci Total Environ 2005; 347:98-105

16. Freire C, Abril A, Fernández MF, et al. Urinary 1-hydroxypyrene and PAH exposure in 4-year-old Spanish children. Sci Total Environ 2009:407:1562-9.

17. Tuntawiroon J, Mahidol C, Navasumrit $P$, et al. Increased health risk in Bangkok children exposed to polycyclic aromatic hydrocarbons from traffic-related sources. Carcinogenesis 2007:28:816-22

18. Martínez-Salinas RI, Elena Leal M, Batres-Esquivel LE, et al. Exposure of children to polycyclic aromatic hydrocarbons in Mexico: assessment of multiple sources. Int Arch Occup Environ Health 2010;83:617-23.

19. Hu SW, Chan YJ, Hsu HT, et al. Urinary levels of 1-hydroxypyrene in children residing near a coal-fired power plant. Environ Res 2011:111:1185-91.

20. Hecht S. Carcinogen metabolites as biomarkers. In: Wild C, Vineis P, Garte S, eds. Molecular Epidemiology of Chronic Diseases. West Sussex, UK, 2008:97-110.
21. Merlo F, Bolognesi C, Peluso M, et al. Airborne levels of polycyclic aromatic hydrocarbons: 32P-postlabeling DNA adducts and micronuclei in white blood cells from traffic police workers and urban residents. J Environ Pathol Toxicol Oncol 1997; 16:157-62.

22. Topinka J, Sevastyanova O, Binkova B, et al. Biomarkers of air pollution exposure-a study of policemen in Prague. Mutat Res 2007:624:9-17.

23. Perera F, Brenner D, Jeffrey A, et al. DNA adducts and related biomarkers in populations exposed to environmental carcinogens. Environ Health Perspect 1992;98:133-7.

24. Hemminki K, Zhang LF, Krüger $\mathrm{J}$, et al. Exposure of bus and taxi drivers to urban air pollutants as measured by DNA and protein adducts. Toxicol Lett 1994; 72:171-4.

25. Nielsen PS, de Pater $\mathrm{N}$, Okkels $\mathrm{H}$, et al. Environmental air pollution and DNA adducts in Copenhagen bus drivers-Effect of GSTM1 and NAT2 genotypes on adduct levels. Carcinogenesis 1996:17:1021-7.

26. Nielsen PS, Okkels H, Sigsgaard T, et al. Exposure to urban and rural air pollution: DNA and protein adducts and effect of glutathione-S-transferase genotype on adduct levels. Int Arch Occup Environ Health 1996;68:170-6.

27. Ayi-Fanou L, Avogbe PH, Fayomi B, et al. DNA-adducts in subjects exposed to urban air pollution by benzene and polycyclic aromatic hydrocarbons (PAHs) in Cotonou, Benin. Environ Toxicol 2011;26:93-102

28. Whyatt RM, Santella RM, Jedrychowski W, et al. Relationship between ambient air pollution and DNA damage in Polish mothers and newborns. Environ Health Perspect 1998;106(Suppl 3):821-6.

29. Topinka J, Binkova B, Mrackova G, et al. DNA adducts in human placenta as related to air pollution and to GSTM1 genotype. Mutat Res 1997;390:59-68.

30. Herbstman JB, Tang D, Zhu D, et al. Prenatal exposure to polycyclic aromatic hydrocarbons, Benzo[a]Pyrene-DNA adducts and genomic DNA methylation in cord blood. Environ Health Perspect. Published Online First: 17 January 2012. doi:10.1289/ ehp.1104056

31. Sørensen M, Autrup $\mathrm{H}$, Hertel 0 , et al. Personal exposure to PM2.5 and biomarkers of DNA damage. Cancer Epidemiol Biomark Prev 2003;12:191-6.

32. Yang K, Airoldi L, Pastorelli R, et al. Aromatic DNA adducts in lymphocytes of humans working at high and low traffic density areas. Chem Biol Interact 1996;101:127-36.

33. Peluso M, Munnia A, Palli D, et al. Bulky DNA adducts and lung cancer risk: a prospective study in EPIC investigation. AACR Meeting Abstracts 2005; 2005:512-a.

34. Palli D, Vineis P, Russo A, et al. Diet, metabolic polymorphisms and DNA adducts: the EPIC-Italy cross-sectional study. Int J Cancer 2000;87:444-51.

35. Veglia F, Loft S, Matullo G, et al. DNA adducts and cancer risk in prospective studies: a pooled analysis and a meta-analysis. Carcinogenesis 2008;29:932-6.

36. Cooke MS, Olinski R, Loft $S$. Measurement and meaning of oxidatively modified DNA lesions in urine. Cancer Epidemiol Biomark Prev 2008:17:3-14.

37. Vinzents PS, Møller P, Sørensen M, et al. Personal exposure to ultrafine particles and oxidative DNA damage. Environ Health Perspect 2005:113:1485-90.

38. Bräuner EV, Forchhammer L, Møller $P$, et al. Exposure to ultrafine particles from ambient air and oxidative stress-induced DNA damage. Environ Health Perspect 2007; 115:1177-82

39. Danielsen PH, Bräuner EV, Barregard L, et al. Oxidatively damaged DNA and its repair after experimental exposure to wood smoke in healthy humans. Mutat Res 2008;642:37-42

40. Suzuki J, Inoue Y, Suzuki S. Changes in the urinary excretion level of 8-hydroxyguanine by exposure to reactive oxygen-generating substances. Free Radic Biol Med 1995; 18:431-6.

41. Allen J, Trenga CA, Peretz A, et al. Effect of diesel exhaust inhalation on antioxidant and oxidative stress responses in adults with metabolic syndrome. Inhal Toxicol 2009;21:1061-7.

42. Chuang KJ, Chan CC, Su TC, et al. The effect of urban air pollution on inflammation oxidative stress, coagulation, and autonomic dysfunction in young adults. Am J Respir Crit Care Med 2007;176:370-6.

43. Kim JY, Prouty LA, Fang SC, et al. Association between fine particulate matter and oxidative DNA damage may be modified in individuals with hypertension. J Occup Environ Med 2009;51:1158-66.

44. Avogbe PH, Ayi-Fanou L, Autrup H, et al. Ultrafine particulate matter and high-level benzene urban air pollution in relation to oxidative DNA damage. Carcinogenesis 2005; 26:613-20.

45. De Coster S, Koppen G, Bracke M, et al. Pollutant effects on genotoxic parameters and tumor-associated protein levels in adults: a cross sectional study. Environ Health 2008; 7:26.

46. Sorensen M, Skov $\mathrm{H}$, Autrup $\mathrm{H}$, et al. Urban benzene exposure and oxidative DNA damage: influence of genetic polymorphisms in metabolism genes. Sci Total Environ 2003:309:69-80.

47. Lagorio S, Tagesson C, Forastiere F, et al. Exposure to benzene and urinary concentrations of 8-hydroxydeoxyguanosine, a biological marker of oxidative damage to DNA. Occup Environ Med 1994:51:739-43.

48. Møller $\mathbf{P}$, Loft S. Oxidative damage to DNA and lipids as biomarkers of exposure to air pollution. Environ Health Perspect 2010;118:1126-36.

49. Møller $\mathbf{P}$, Folkmann JK, Forchhammer L, et al. Air pollution, oxidative damage to DNA, and carcinogenesis. Cancer Lett 2008;266:84-97. 
50. Knudsen LE, Norppa H, Gamborg MO, et al. Chromosomal aberrations in humans induced by urban air pollution: influence of DNA repair and polymorphisms of glutathioneS-transferase M1 and N-acetyltransferase 2. Cancer Epidemiol Biomark Prev 1999:8:303-10.

51. Srám RJ, Binková $B$, Rössner $P$, et al. Adverse reproductive outcomes from exposure to environmental mutagens. Mutat Res 1999;428:203-15.

52. Burgaz S, Cakmak Demircigil G, Karahalil B, et al. Chromosomal damage in peripheral blood lymphocytes of traffic policemen and taxi drivers exposed to urban air pollution. Chemosphere 2002:47:57-64.

53. Sram RJ, Beskid 0, Rössnerova A, et al. Environmental exposure to carcinogenic polycyclic aromatic hydrocarbons: the interpretation of cytogenetic analysis by FISH. Toxicol lett 2007:172:12-20.

54. Balachandar V, Kumar BL, Suresh K, et al. Evaluation of chromosome aberrations in subjects exposed to environmental tobacco smoke in Tamilnadu, India. Bull Environ Contam Toxicol 2008;81:270-6.

55. Rossner P Jr, Uhlirova K, Beskid 0, et al. Expression of XRCC5 in peripheral blood lymphocytes is upregulated in subjects from a heavily polluted region in the Czech Republic. Mutat Res 2011;713:76-82.

56. Rossnerova A, Spatova M, Pastorkova A, et al. Micronuclei levels in mothers and their newborns from regions with different types of air pollution. Mutat Res 2011:715:72-8.

57. García-Suástegui WA, Huerta-Chagoya A, Carrasco-Col'n KL, et al. Seasonal variations in the levels of PAH-DNA adducts in young adults living in Mexico City. Mutagenesis 2011;26:385-91.

58. Kyrtopoulos SA, Georgiadis P, Autrup H, et al. Biomarkers of genotoxicity of urban air pollution: overview and descriptive data from a molecular epidemiology study on populations exposed to moderate-to-low levels of polycyclic aromatic hydrocarbons: the AULIS project. Mutat Res 2001;496:207-28.
59. Zidzik J, Kalina I, Salagovic J, et al. Influence of PAHs in ambient air on chromosomal aberrations in exposed subjects: international study-EXPAH. Mutat Res 2007;620:41-8.

60. Micronucleus. 2010. http://ntp.niehs.nih.gov/?objectid=16D65516-BA99-8D3EBEFF712372F4B675 (accessed 16 Nov 2010).

61. Pedersen M, Wichmann J, Autrup $\mathrm{H}$, et al. Increased micronuclei and bulky DNA adducts in cord blood after maternal exposures to traffic-related air pollution. Environ Res 2009;109:1012-20.

62. Ishikawa H, Tian Y, Piao F, et al. Genotoxic damage in female residents exposed to environmental air pollution in Shenyang city, China. Cancer Lett 2006;240:29-35

63. Neri M, Ugolini D, Bonassi S, et al. Children's exposure to environmental pollutants and biomarkers of genetic damage. II. Results of a comprehensive literature search and meta-analysis. Mutat Res 2006;612:14-39.

64. Lechner M, Boshoff C, Beck S. Cancer epigenome. Adv Genet 2010;70:247-76.

65. Baccarelli A, Zanobetti A, Martinelli I, et al. Air pollution, smoking, and plasma homocysteine. Environ Health Perspect 2007;115:176-81.

66. Baccarelli A, Wright RO, Bollati V, et al. Rapid DNA methylation changes after exposure to traffic particles. Am J Respir Crit Care Med 2009;179:572-8.

67. Tarantini L, Bonzini M, Apostoli $P$, et al. Effects of particulate matter on genomic DNA methylation content and iNOS promoter methylation. Environ Health Perspect 2009;117:217-22.

68. Madrigano J, Baccarelli A, Mittleman MA, et al. Prolonged exposure to particulate pollution, genes associated with glutathione pathways, and DNA methylation in a cohort of older men. Environ Health Perspect 2011;119:977-82.

69. Alexeeff SE, Coull BA, Gryparis A, et al. Medium-term exposure to traffic-related air pollution and markers of inflammation and endothelial function. Environ Health Perspect. Published Online First: 24 February 2011. doi:10.1289/ehp.1002560 